\title{
Role of Chemical Potentials in Surface Reconstruction: A New Model and Phase Transition of GaAs(111)2x2
}

\section{Citation}

Kaxiras, Efthimios, K. C. Pandey, Yaneer Bar-Yam, and John D. Joannopoulos. Role of chemical potentials in surface reconstruction: A new model and phase transition of GaAs(111)2x2.

Physical Review Letters 56, no. 26: 2819-2821.

\section{Published Version}

http://dx.doi.org/10.1103/PhysRevLett.56.2819

\section{Permanent link}

http://nrs.harvard.edu/urn-3:HUL.InstRepos:2894634

\section{Terms of Use}

This article was downloaded from Harvard University's DASH repository, and is made available under the terms and conditions applicable to Other Posted Material, as set forth at http:// nrs.harvard.edu/urn-3:HUL.InstRepos:dash.current.terms-of-use\#LAA

\section{Share Your Story}

The Harvard community has made this article openly available.

Please share how this access benefits you. Submit a story.

\section{Accessibility}




\title{
Role of Chemical Potentials in Surface Reconstruction: A New Model and Phase Transition on GaAs(111) $2 \times 2$
}

\author{
E. Kaxiras ${ }^{(a)}$ and K. C. Pandey \\ IBM Thomas J. Watson Research Center, Yorktown Heights, New York 10598 \\ and \\ Y. Bar-Yam and J. D. Joannopoulos \\ Department of Physics, Massachusetts Institute of Technology, Cambridge, Massachusetts 02139
}

(Received 18 February 1986)

\begin{abstract}
The role of chemical potentials in surface reconstruction is examined and shown to be crucial for binary semiconductor surfaces such as GaAs(111) $2 \times 2$. We predict that under As-rich conditions a new model, the As triangle, is the lowest-energy geometry, whereas the Ga-vacancy model is appropriate for Ga-rich conditions. A change in the relative chemical potential of $\mathrm{Ga}$ and As should produce a phase transition between the two structures.
\end{abstract}

PACS numbers: $68.35 . \mathrm{Md}, 73.20 . \mathrm{Cw}$

The theoretical studies of semiconductor surface reconstructions do not in general consider the effect of the chemical potential of the atoms. This is justified in the case of elemental semiconductors where, assuming equilibrium with the bulk, the chemical potential is uniquely determined. However, in the case of binarycompound semiconductors the relative chemical potential of the two species of atoms is an independent parameter and can play a crucial role in determining the equilibrium surface configuration.

In this Letter we wish to exhibit the importance of the chemical potentials in surface reconstruction by considering a prototypical binary-semiconductor surface, which has received much attention in the literature lately, ${ }^{1-4}$ namely the (111) surface of GaAs. In particular, we will give a detailed theoretical discussion of the following points.

(i) For surfaces with stoichiometries different from the corresponding bulk atomic plane it is meaningless to simply ask what is the lowest-energy geometry of the surface. It is crucial to include the $\mathrm{Ga}$ and As chemical potentials as defined by the relative abundance of each species during preparation.

(ii) Under As-rich conditions, the lowest-energy $2 \times 2$ reconstruction of $\mathrm{GaAs}(111)$ is a new model, the As-triangle geometry consisting of three As adatoms bonded in a triangular configuration, whereas for a Ga-rich environment the lowest-energy $2 \times 2$ reconstruction is the Ga-vacancy geometry. ${ }^{1,2,5}$ In an Asrich environment the As triangle is lower in energy than the $\mathrm{Ga}$ vacancy by as much as $2.0 \mathrm{eV}$ per $2 \times 2$ surface unit cell.

(iii) Under equilibrium conditions a first-order phase transition should be observable as a function of the relative chemical potential of As and $\mathrm{Ga}$, from the As-triangle to the Ga-vacancy geometry. Thus, even though both models constitute an As-rich surface (due to an excess of As atoms or a deficit of $\mathrm{Ga}$ atoms), the surface preparation conditions play a fundamental role in determining the experimentally observed structure.

Experimentally, the chemical potentials are determined by the surface-preparation conditions. There are two extreme conditions, to which we will refer as the "As-rich" and "Ga-rich" environments. In these environments the majority component interacts with the surface to determine the equilibrium structure. Thus for the As-rich environment, excess As is available in the form of $\mathrm{As}_{2}$ gas, whereas in the Ga-rich environment the $\mathrm{Ga}$ source is metallic bulk gallium. This choice of atomic reservoirs is consistent with the usual preparation conditions described in experimental work. ${ }^{6}$ One final consideration involves annealed surfaces, on which several experiments have been performed. ${ }^{1,3,4}$ Taking into account that the sticking coefficient of As on GaAs surfaces is much lower than that of $\mathrm{Ga}$, we suggest that annealing under highvacuum conditions would simulate a Ga-rich environment. In this case we conclude from the results of experimental ${ }^{1,3,4}$ and theoretical ${ }^{2,5}$ studies that the stable reconstruction is the Ga-vacancy geometry. The fact that this geometry implies an As-rich surface is not incompatible with the assumed Ga-rich environment: The excess $\mathrm{Ga}$ atoms are in equilibrium with $\mathrm{Ga}$ bulk and GaAs bulk, occupying bulk vacancy sites or condensing into surface $\mathrm{Ga}$ droplets.

We now concentrate on the GaAs(111) surface in an As-rich environment, and examine various low-energy geometries. In order to compare the different surface models we calculate the total energy of each configuration at zero temperature. The methodology employed has been described in detail elsewhere. ${ }^{5}$ It suffices to note that the calculations are based on a firstprinciples, self-consistent evaluation of the total ener$\mathrm{gy}$, in the local-density-functional formalism, using the 
pseudopotential approximation for the atomic cores. The standard definition of the chemical potential, in the thermodynamic limit, at zero temperature, reduces to $\mu=\epsilon_{a}-\epsilon_{b}$, where $\epsilon_{a}$ is the total energy of an isolated atom and $\epsilon_{b}$ the binding energy per atom of the atomic reservoir. In the context of the present work the total energies of isolated As and $\mathrm{Ga}$ atoms were calculated in the manner described above. The binding energies of particular reservoirs were either calculated $^{7}$ ( $6.8 \mathrm{eV}$ per pair for GaAs bulk) or taken from tabulated values ${ }^{8}\left(2.0 \mathrm{eV}\right.$ per atom for $\mathrm{As}_{2}$ gas, $2.8 \mathrm{eV}$ per atom for $\mathrm{Ga}$ bulk). The chemical potentials of atoms added to (or removed from) a given surface configuration were added to (or subtracted from) the calculated total energy of this configuration. Thus the total number of atoms in the system is kept constant. This approach enables us to include the chemical potentials of the constituent atoms in the energy comparisons in a consistent way.

The energies of the different models in As-rich conditions (excess $\mathrm{As}_{2}$ gas), with respect to the ideal unreconstructed surface, are displayed in Fig. 1. The Ga-vacancy configuration (with simultaneous creation of GaAs bulk pairs) is lower in energy than the Asadatom configuration by $2.3 \mathrm{eV}$ per $2 \times 2$ unit cell. This is due to the unfavorable geometric coordination of the As adatom which forms three Ga-As surface bonds tilted with respect to the corresponding vertical GaAs bulk bonds. The As-triangle model is lower in energy than the ideal surface plus $\frac{3}{2} \mathrm{As}_{2}$ molecules by $5.1 \mathrm{eV}$ per $2 \times 2$ unit cell and lower by $2.0 \mathrm{eV}$ than the Ga-vacancy model. The primary reason for the low energy of this model is that the three As adatoms are bonded through As-As bonds which should effectively compensate for the energy required to dissociate
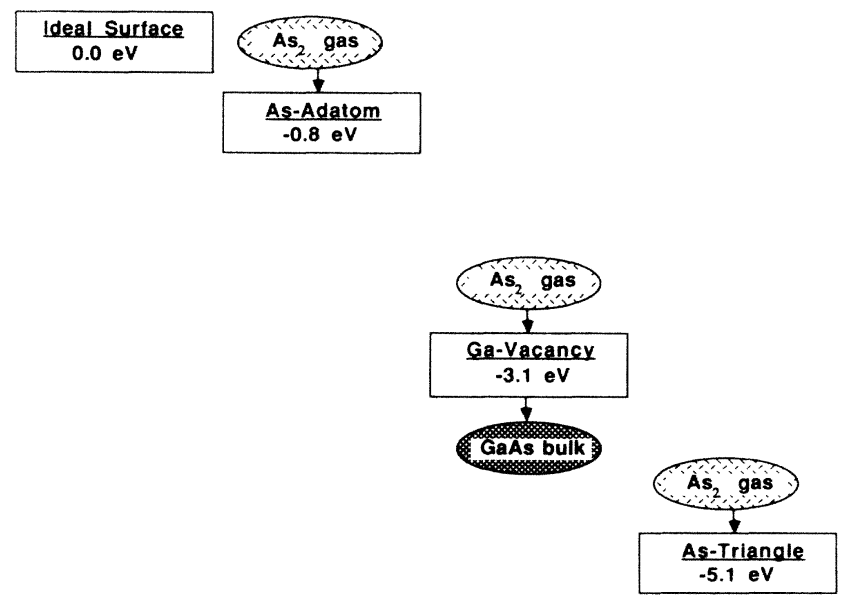

FIG. 1. Total energy in electronvolts per $2 \times 2$ unit cell for various reconstructions of the (111) surface of $\mathrm{GaAs}$ under As-rich conditions. the $\mathrm{As}_{2}$ molecules which are the source of excess As atoms. An additional reason contributing to the low energy of the As triangle is the nearly perfect geometric coordination of the remaining surface As and $\mathrm{Ga}$ atoms, which we now describe.

The three As adatoms per $2 \times 2$ unit cell form three bonds with surface $\mathrm{Ga}$ atoms and three As-As bonds in a triangular configuration (Fig. 2). The remaining Ga surface dangling bond is empty as a result of relaxation and rehybridization: The fourth surface $\mathrm{Ga}$ atom recedes toward the bulk and becomes threefold coordinated, almost planar with the three neighboring As atoms. This atomic arrangement allows the As atoms in the triangle to relax with respect to each other as well as with respect to the surface Ga atoms to which they are bonded. The three $\mathrm{Ga}$-As surface bonds are not tilted by the same amount as those of the single As adatom, and therefore are closer to the GaAs bulk bonds. The As-As bonds have enough phase space to achieve optimal bonding, the only constraint being that they form three $60^{\circ}$ angles between them. This constraint is a result of the equilateral triangle arrangement of the As atoms. Since the surface Ga atoms to which the As triangle binds are equivalent by symmetry, there is no symmetry-breaking feature on the surface to suggest departure from the equilateral triangle configuration.

Through our energy-minimization calculations we determine that in the optimal configuration the threefold-coordinated As adatoms form bonds with average angular separation $91.7^{\circ}$. The surface Ga-As
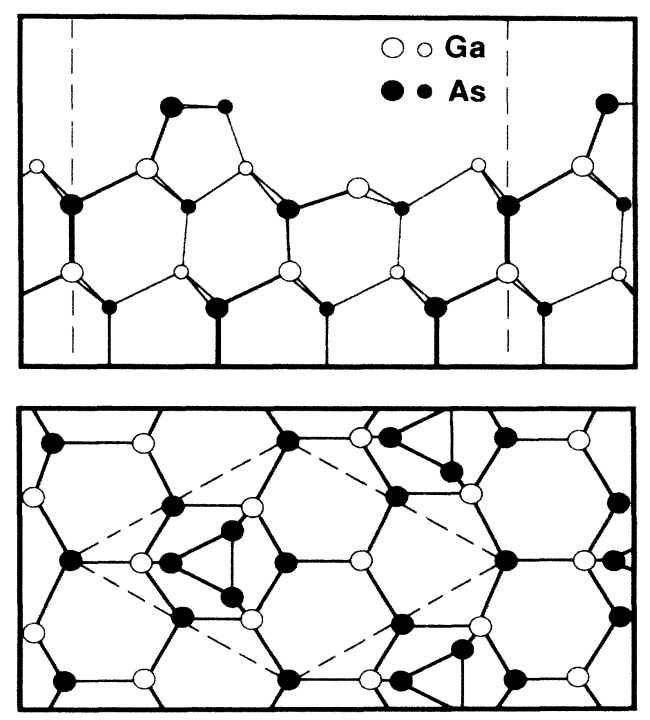

FIG. 2. Upper panel: Side view [(110) plane] of the GaAs(111) $2 \times 2$ surface As triangle model. Larger size atoms are in the plane of the paper. Smaller size atoms are in a parallel plane. Lower panel: Top view of the Astriangle model. Dashed lines indicate a $2 \times 2$ unit cell. 
bonds are equal in bond length (to within $0.1 \%$ ) to the bulk GaAs bonds. The charge density of the first five atomic layers (counting from the surface downward) is shown in Fig. 3 (upper panel). The plane of this figure is the (110) plane of GaAs. From this charge density, the bond between the surface As atom and the nearest $\mathrm{Ga}$ atom is seen to be similar in nature to bulk GaAs bonds. The As-As bonds have a bond length of $2.47 \AA$ ( $1.5 \%$ shorter than that of As bulk). These bonds are exhibited in Fig. 3 (lower panel) which shows the charge density on a plane passing through the As triangle. They are not considerably different from bulk GaAs bonds. In fact, the highest chargedensity contour in the As-As bond has the same value as that in a bulk bond. Finally, the fourth surface $\mathrm{Ga}$ atom forms three bonds shorter by $1.0 \%$ than the ideal GaAs bond and three angles of $114.7^{\circ}$. The atoms in the first bilayer under the surface are also relaxed by small amounts relative to their ideal positions to relieve some of the strain introduced by the surface relaxation.

Let us now consider the possibility of a phase transition as a function of the chemical potentials. Our results suggest that by varying the relative chemical potential of As and $\mathrm{Ga}$ a phase transition should occur between the As-triangle and Ga-vacancy geometries as the conditions are changed from As rich to $\mathrm{Ga}$ rich, respectively. This is consistent with the observation of Cho and Arthur ${ }^{9}$ that the intensity of the half-order LEED spots varies as the ratio of $\mathrm{As}_{2}$ to $\mathrm{Ga}$ is changed on the (111) surface, although the overall pattern
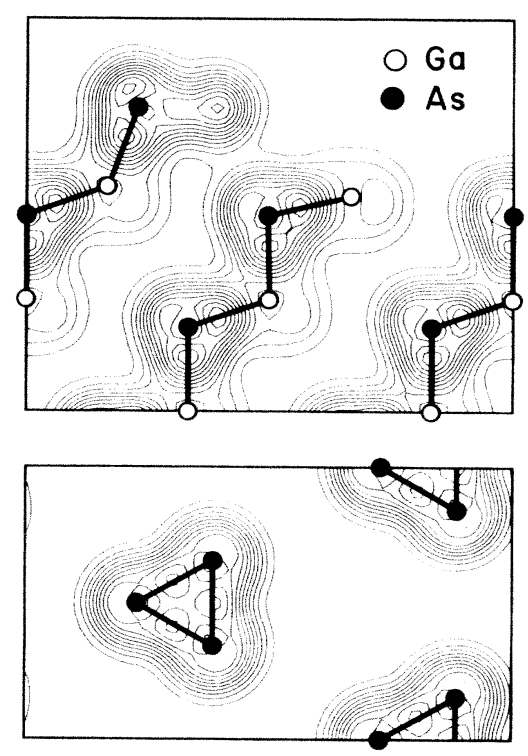

FIG. 3. Upper panel: Charge density of the GaAs(111)$2 \times 2$ As-triangle model in the (110) plane, passing through one of the As-triangle atoms. Lower panel: Charge density of As-triangle model on the plane of the As triangle. remains $2 \times 2$. Since the Ga vacancy and the As triangle are two different and unrelated structures, we expect the transition between them to be discontinuous and thus first order, at least in the $T=0 \mathrm{~K}$ case. This should be true even at finite temperature, as long as the transition temperature is much lower than the free-energy difference between the two structures. Given the large energy difference of the two geometries at $T=0 \mathrm{~K}$, and assuming that the relevant entropies do not change this difference significantly, we expect the transition to remain first order at $T$ $>0 \mathrm{~K}$.

An estimate of the chemical potential at which the transition should occur can be obtained by considering the relevant $\mathrm{Ga}$ and As chemical potentials at zero temperature. Equilibrium with bulk GaAs implies that the two chemical potentials must add up to the value of the GaAs cohesive energy. This indicates that the phase transition will occur at an As chemical potential lower by approximately $1.0 \mathrm{eV}$ from its value in the As-rich environment. The change in chemical potential can be achieved experimentally by varying the partial pressures of $\mathrm{Ga}$ and $\mathrm{As}$, or the temperature at which the sample is prepared. However, the value mentioned above is only a rough estimate, since our present calculations are concerned with the zerotemperature conditions. A proper comparison with experiment must include finite-temperature effects. We are currently involved in extending this study to the finite-temperature regime.

This work was supported in part by Joint Services Electronics Program, Department of Defense Contract No. DAAL 03-86-K-0002. One of us (E.K) would like to thank the IBM Thomas J. Watson Research Center for its hospitality while portions of this work were carried out, and acknowledge support by an IBM PreDoctoral Fellowship. One of us (Y.B.) would like to acknowledge support by a Bantrell Post-Doctoral Fellowship.

\footnotetext{
(a) Permanent address: Department of Physics, Massachusetts Institute of Technology, Cambridge, Mass. 02139.

1S. Y. Tong, G. Xu, and W. N. Mei, Phys. Rev. Lett. 52, 1693 (1984).

2D. J. Chadi, Phys. Rev. Lett. 52, 1911 (1984).

${ }^{3}$ R. D. Bringans and R. Z. Bachrach, Phys. Rev. Lett. 53, 1954 (1984).

4J. Bohr, R. Feidenhans'1, M. Nielsen, M. Toney, R. L. Johnson, and I. K. Robinson, Phys. Rev. Lett. 54, 1275 (1985).
}

${ }^{5}$ E. Kaxiras, Y. Bar-Yam, J. D. Joannopoulos, and K. C. 
Pandey, Phys. Rev. B 33, 4406 (1986).

${ }^{6}$ A. Y. Cho and I. Hayashi, Solid State Electron. 14, 125 (1971).

${ }^{7}$ This result was obtained through a bulk GaAs pseudopotential calculation using the same potentials and energy cutoffs as the present work. It is in good agreement with exper- iment $(6.7 \mathrm{eV}$ per GaAs pair).

${ }^{8} \mathrm{R}$. Hultgren et al., Selected Values of the Thermodynamic Properties of the Elements (American Society for Metals, Metals Park, Ohio, 1973).

${ }^{9}$ A. Y. Cho and J. R. Arthur, Prog. Solid State Chem. 10, $157(1975)$. 